\title{
THE FIXED-POINT CONSTRUCTION IN EQUIVARIANT BORDISM
}

BY

\author{
RUSSELL J. ROWLETT
}

\begin{abstract}
Consider the bordism $\Omega_{*}(G)$ of smooth $G$-actions. If $K$ is a subgroup of $G$, with normalizer $N K$, there is a standard $N K / K$-action on $\Omega_{*}(K)$ (All, Proper). If $M$ has a smooth $G$-action, a tubular neighborhood of the fixed set of $K$ in $M$ represents an element of $\Omega_{*}(K)$ (All, Proper) ${ }^{N K / K}$. One thus obtains the "fixed point homomorphism" $\phi$ carrying $\Omega_{*}(G)$ to the sum of the $\Omega_{*}(K)$ (All, Proper) ${ }^{N K / K}$, summed over conjugacy classes of subgroups $K$. Let $P$ be the collection of primes not dividing the order of $G$. We show that the $P$-localization of $\phi$ is an isomorphism, and give several applications.
\end{abstract}

1. The fixed-point homomorphism. Let $G$ be a finite group. We shall be concerned with smooth actions of $G$ on compact manifolds $M$, preserving either an orientation or a unitary structure.

The notation is based on that of Stong [8], [9]. The unadorned symbol $\Omega_{*}$ denotes either the oriented bordism ring $\Omega_{*}^{s O}$ or the unitary bordism ring $\Omega_{*}^{U}$. If $K$ is a subgroup of $G$, then $A K$ is the family of all subgroups of $G$ conjugate to subgroups of $K$, and $P K$ is the family of subgroups conjugate to proper subgroups of $K$. Thus $\Omega_{*}(G)$ or $\Omega_{*}(G)(A G)$ is the bordism of all $G$-actions, while $\Omega_{*}(G)(A G, P G)$ is the bordism of all $G$-actions on manifolds with boundary, such that each point $x$ of $\partial M$ has isotropy subgroup $G_{x}$ in $P G$.

Beginning with the famous monograph of Conner and Floyd [2], most research in equivariant bordism has involved fixed-point constructions of the following sort. Let $K$ be a subgroup of $G$. If $M$ is a closed manifold with a smooth $K$-action, then the fixed set of $K$ in $M$ has a $K$-invariant tubular neighborhood $N$. Assigning $N$ to $M$ defines a homomorphism $f_{K}: \Omega_{*}(K) \rightarrow$ $\Omega_{*}(K)(A K, P K)$, which is of interest because the "relative" group $\Omega_{*}(K)(A K, P K)$ is generally easier to compute than is $\Omega_{*}(K)$. If $r_{K}^{G}: \Omega_{*}(G) \rightarrow$ $\Omega_{*}(K)$ is the forgetful homomorphism restricting $G$-actions to $K$-actions, there is the composition $f_{K} r_{K}^{G}: \Omega_{*}(G) \rightarrow \Omega_{*}(K)(A K, P K)$.

Definition. If $K$ is a subgroup of $G$, let $(K)$ be the set of subgroups conjugate to $K$ in $G$. If $\mathscr{F}^{\prime} \subseteq \mathscr{F}$ are families of subgroups of $G$, in the sense of

Received by the editors September 19, 1977.

AMS (MOS) subject classifications (1970). Primary 57D85.

Key words and phrases. Finite group actions, equivalent bordism, fixed point homomorphism.

(c) American Mathematical Society 1979 
[9], the fixed point homomorphism

$$
\phi: \Omega_{*}(G)\left(\mathscr{F}, \mathscr{F}^{\prime}\right) \rightarrow \sum\left\{\Omega_{*}(K)(A K, P K):(K) \subset \mathscr{F}-\mathscr{F}^{\prime}\right\}
$$

is the sum of the homomorphisms $f_{K} r_{K}^{G}$, one for each conjugacy class of subgroups in $\mathscr{F}-\mathscr{F}^{\prime}$.

It is convenient to reduce the range of $\phi$ to a submodule of "invariant" elements. Let $N(K)$ be the normalizer of $K$ in $G$. The quotient group $L=N(K) / K$ acts on $\Omega_{*}(K)(A K, P K)$ as follows: given a smooth $K$-action $\theta: K \times M \rightarrow M$ and an element $g \in G$, there is a new $K$-action $g_{*} \theta$ on $M$ defined by the rule $g_{*} \theta(k, m)=\theta\left(g^{-1} k g, m\right)$. If $\bar{g}$ is the coset $g K$ in $L$, the formula $\bar{g}[M, \theta]=\left[M, g_{*} \theta\right]$ gives a well-defined $L$-action by automorphisms of $\Omega_{*}(K)(A K, P K)$. Let $\Omega_{*}(K)(A K, P K)^{N(K) / K}$ be the submodule fixed elementwise under this action. It follows from $[2, \S 20]$ that the image of $\phi$ is a submodule of $\Sigma_{(K)} \Omega_{*}(K)(A K, P K)^{N(K) / K}$.

If $P$ is a collection of prime numbers, $Z_{P}$ is the ring of $P$-local integers. In particular, if $P$ is the set of all odd primes $Z_{P}$ is the subring $Z\left(\frac{1}{2}\right)$ of the rationals, generated by $Z$ and $\frac{1}{2}$. (The notation $Z_{m}$, with lower case subscript, is reserved for the cyclic group of order $m$.) Let $P(G)$ be the collection of primes which do not divide the order of $G$.

THEOREM 1.1. Suppose $G$ is a finite group and $P=P(G)$. Then the localized fixed point homomorphism,

$$
\phi_{P}: \Omega_{*}(G) \otimes Z_{P} \rightarrow \sum_{(K)} \Omega_{*}(K)(A K, P K)^{N(K) / K} \otimes Z_{P},
$$

is an isomorphism.

In particular, the kernel of $\phi$ is a torsion group. Combining Theorem 1.1 with [5, Theorem 1] yields the following.

COROLlary 1.2. Suppose $G$ is a finite abelian group. Then

$$
\phi: \Omega_{*}^{U}(G) \rightarrow \sum_{(K)} \Omega_{*}^{U}(K)(A K, P K)
$$

is a monomorphism. The analogous theorem for $\Omega_{*}^{S O}$ holds if $G$ is abelian of odd order.

Another corollary of the theorem is Wheeler's theorem [11] that $\Omega_{*}^{U}(G) \otimes$ $Z_{P}$ is a free $\Omega_{*}^{U} \otimes Z_{P}$-module on even-dimensional generations. However, the present proof is much more elementary than Wheeler's, so much so that the method can be transferred very readily to other bordism theories. Hopefully, Theorem 1.1 will thus be a source of new insight into the workings of equivariant bordism.

I would like to thank R. E. Stong for pointing out several errors in an early 
version of this paper, and the University of Tennessee Faculty Research Fund for financial support during a portion of the time that the paper was in preparation.

2. A spectral sequence. Suppose $K$ is a normal subgroup of $G$. A family $\mathscr{F}$ of subgroups of $K$ is $G$-invariant if $g \mathrm{Hg}^{-1} \in \mathscr{F}$ whenever $H \in \mathscr{F}$, for every $g \in G$. If $\left(\mathscr{F}, \mathscr{F}^{\prime}\right)$ is a pair of $G$-invariant families of subgroups of $K$, then the quotient group $L=G / K$ acts on $\Omega_{*}(K)\left(\mathscr{F}, \mathscr{F}^{\prime}\right)$, just as in the previous section.

Proposition 2.1. Suppose $K$ is a normal subgroup of $G, L=G / K$, and $\left(\mathscr{F}, \mathscr{F}^{\prime}\right)$ is a pair of $G$-invariant families of subgroups of $K$. Let $\Omega_{*}(K)\left(\mathscr{F}, \mathscr{F}^{\prime}\right)$ be an $L$-module as above. Then there is a first quadrant spectral sequence $\left\{E^{r}=\right.$ $\left.E^{r}(G, K)\left(\mathscr{F}, \mathscr{F}^{\prime}\right): r \geqslant 2\right\}$ such that

(a) $E_{a, b}^{2} \cong H_{a}\left(L ; \Omega_{b}(K)\left(\mathscr{F}, \mathscr{F}^{\prime}\right)\right)$;

(b) $E^{\infty}$ is associated to a filtration of $\Omega_{*}(G)\left(\mathscr{F}, \mathscr{F}^{\prime}\right)$; and

(c) the edge homomorphism

$$
\Omega_{b}(K)\left(\mathscr{F}, \mathscr{F}^{\prime}\right) \rightarrow E_{0, b}^{2} \rightarrow E_{0, b}^{\infty} \rightarrow \Omega_{b}(G)\left(\mathscr{F}, \mathscr{F}^{\prime}\right)
$$

is the extension homomorphism, $e_{G}^{K}$.

Proof. Begin with a classifying space $E L$ for principal $L$-bundles. We may assume, for example via the Milnor construction [4], that $E L$ is a CW-complex in such a way that its $n$-skeleton $E L^{n}$ is obtained from $E L^{n-1}$ by attaching a finite number of copies of $L \times D^{n}$, in which $L$ acts trivially on $D^{n}$. The projection $G \rightarrow L$ makes $E L$ a $G$-space.

If $M$ has a smooth $G$-action, all of whose isotropy groups lie in $K$, then the quotient space $M / K$ inherits a free action of $L$, and thus admits an $L$-equivariant map $h: M / K \rightarrow E L$. The composition $M \rightarrow M / K \stackrel{h}{\rightarrow} E L$ is then unique, up to a $G$-equivariant homotopy. This observation implies that $\Omega_{*}(G)\left(\mathscr{F}, \mathscr{F}^{\prime}\right) \cong \Omega_{*}(G)\left(\mathscr{F}, \mathscr{F}^{\prime}\right)(E L)$.

By the usual considerations, there is now a spectral sequence $\left\{E^{r}: r \geqslant 1\right\}$ such that $E_{a, b}^{1}=\Omega_{a+b}(G)\left(\mathscr{F}, \mathscr{F}^{\prime}\right)\left(E L^{a}, E L^{a-1}\right)$ and $d_{a, b}^{1}$ is equal to the boundary homomorphism of the triple $\left(E L^{a}, E L^{a-1}, E L^{a-2}\right)$.

The rest is an easy calculation. If $X$ is obtained from $A$ by attaching $L \times D^{n}$ then

$$
\Omega_{k}(G)\left(\mathscr{F}, \mathscr{F}^{\prime}\right)(X, A) \cong \Omega_{k-n}(K)\left(\mathscr{F}, \mathscr{F}^{\prime}\right) .
$$

This is obvious since bordism is a $G$-homology theory [8, Proposition (2.1)]. It follows that

$$
\Omega_{a+b}(G)\left(\mathscr{F}, \mathscr{F}^{\prime}\right)\left(E L^{a}, E L^{a-1}\right) \cong H_{a}\left(E L^{a}, E L^{a-1}\right) \otimes_{L} \Omega_{b}(K)\left(\mathscr{F}, \mathscr{F}^{\prime}\right),
$$

so that $d_{a, b}^{1}$ corresponds to $\partial \otimes 1$, where $\partial$ is the boundary homomorphism of 
$\left(E L^{a}, E L^{a-1}, E L^{a-2}\right)$ in ordinary homology. This proves (a). The construction is such that (b) and (c) are built in, if we begin with $E L^{0}=L$

If $K$ is not central in $G$, then the coefficients of the $E^{2}$ term are not trivial and the computation of the spectral sequence may be very difficult. However, there is interesting information immediately available. Let $P(G: K)$ be the collection of primes not dividing the index of $K$ in $G$.

Proposition 2.2. Suppose $K$ is normal in $G$, and $P=P(G: K)$. Then the extension,

$$
e_{G}^{K}: \Omega_{*}(K)\left(\mathscr{F}, \mathscr{F}^{\prime}\right) \otimes Z_{P} \rightarrow \Omega_{*}(G)\left(\mathscr{F}, \mathscr{F}^{\prime}\right) \otimes Z_{P},
$$

is an epimorphism for each pair $\left(\mathscr{F}, \mathscr{F}^{\prime}\right)$ of $G$-invariant families of subgroups of $K$. If $K$ is central in $G$, then (1) is an isomorphism.

Proof. If $a>0$ and $S$ is any $L$-module, then $H_{a}(G / K ; S)$ consists entirely of torsion annihilated by $P$-localization. Let $S=\Omega_{b}(K)\left(\mathscr{F}, \mathscr{F}^{\prime}\right)$; then the edge homomorphism (Proposition 2.1c) is a $P$-epimorphism, as required. If $K$ is central, then by [2, p. 54] we know $r_{K}^{G} e_{G}^{K}$ is multiplication by the index of $K$ in $G$. Thus $r_{K}^{G} e_{G}^{K}$, and hence $e_{G}^{K}$, is a $P$-monomorphism.

However, we wish to know about restriction rather than extension.

Proposition 2.3. Let $K$ be a subgroup of $G, P=P(G: K)$, and suppose $\left(\mathscr{F}, \mathscr{F}^{\prime}\right)$ is a pair of $G$-invariant families of subgroups of $K$. Then restriction,

$$
r_{K}^{G}: \Omega_{*}(G)\left(\mathscr{F}, \mathscr{F}^{\prime}\right) \otimes Z_{P} \rightarrow \Omega_{*}(K)\left(\mathscr{F}, \mathscr{F}^{\prime}\right) \otimes Z_{P},
$$

is a monomorphism if and only if (1) is an epimorphism, and (2) is an epimorphism if and only if (1) is a monomorphism.

Proof. Notice that $K$ is not required to be normal. Let $k$ be the index of $K$ in $G$, and write $r$ and $e$ for $r_{K}^{G}$ and $e_{G}^{K}$, respectively. The composition $r e$ is given by the formula

$$
r e(x)=\sum\{\bar{g} x: \bar{g} \in G / K\} \text { for } x \in \Omega_{*}(K)\left(\mathscr{F}, \mathcal{F}^{\prime}\right),
$$

by [2, pp. 53-54]. It follows that ere $(x)=k e(x)$, since $G$ acts trivially on $\Omega_{*}(G)\left(\mathscr{F}, \mathscr{F}^{\prime}\right)$.

Suppose $e$ is a $P$-epimorphism. If $y \in \Omega_{*}(G)\left(\mathscr{F}, \mathscr{F}^{\prime}\right) \otimes Z_{P}$, let $y=e(x)$. If $r(y)=0$, then $k y=k e(x)=\operatorname{ere}(x)=e r(y)=0$, so $y=0$. Thus $r$ is $P$ injective. Now suppose $e$ is a $P$-monomorphism and $x \in \Omega_{*}(K)\left(\mathscr{F}, \mathscr{F}^{\prime}\right) \otimes$ $Z_{P}$; then $e(r e(x)-k x)=0$, which implies that $x=r e(x / k)$ since $e$ is $P$-injective. Thus $r$ is $P$-surjective.

The reader is invited to prove the converse statements, using [9, Proposition 13.2] to compute the composition er.

We shall not require the next proposition, but it is worth mentioning as another easy consequence of Proposition 2.1. 
Proposition 2.4. Suppose $\Omega_{*}(K)\left(\mathscr{F}, \mathscr{F}^{\prime}\right)$ is a free $\Omega_{*}$-module. Then Cartesian product induces an isomorphism

$$
\hat{\Omega}_{*}(L) \otimes_{\Omega_{*}} \Omega_{*}(K)\left(\mathscr{F}, \mathscr{F}^{\prime}\right) \rightarrow \Omega_{*}(L \times K)\left(\mathscr{F}, \mathscr{F}^{\prime}\right) .
$$

In particular, if $K$ is abelian then

$$
\hat{\Omega}_{*}^{U}(L) \otimes_{\Omega_{*}^{U}} \Omega_{*}^{U}(K) \simeq \Omega_{*}^{U}(L \times K)(A K) .
$$

Proof. As in [8], $\hat{\Omega}_{*}(L)$ is the bordism of free $L$-actions. Let $\left\{Q^{r}: r>2\right\}$ be the Atiyah-Hirzebruch spectral sequence, $Q_{a, b}^{2}=H_{a}\left(L: \Omega_{b}\right) \Rightarrow \hat{\Omega}_{*}(L)$, of [2, \$7 and Theorem (19.1)]. From our definition of $E^{r}(L \times K, K)\left(\mathscr{F}, \mathscr{F}^{\prime}\right)$, one clearly has an isomorphism $Q_{a, b}^{2} \otimes_{\Omega_{*}} \Omega_{*}(K)\left(\mathscr{F}, \mathscr{F}^{\prime}\right) \rightarrow E_{a, b}^{2}$ commuting with the differentials, and the proposition then follows immediately.

3. Freeness of localized bordism. Let $\left(\mathscr{F}, \mathscr{F}^{\prime}\right)$ be a pair of families of $G$. If $\mathscr{F}-\mathscr{F}^{\prime}=(K)$, then $\mathscr{F}$ and $\mathcal{F}^{\prime}$ are said to be adjacent, differing by $K$ (see $[9$, p. 19]). In this case, and if $N K$ is the normalizer of $K$ in $G$, then it is known [9, Corollary 5.1, p. 20] that $e_{G}^{N K}$ is an isomorphism of $\Omega_{*}(N K)(A K, P K)$ with $\Omega_{*}(G)\left(\mathscr{F}, \mathscr{F}^{\prime}\right)$. By Proposition 2.3, if $P=P(G: N K)$ the $P$-localization of $r_{N K}^{G}$ is also an isomorphism.

Proposition 3.1. Suppose $\mathscr{F}$ and $\mathscr{F}^{\prime}$ are adjacent, differing by $K$, and $P=P(G: K)$. Then the restriction,

$$
r_{K}^{G}: \Omega_{*}(G)\left(\mathscr{F}, \mathscr{F}^{\prime}\right) \otimes Z_{P} \rightarrow \Omega_{*}(K)(A K, P K)^{N K / K} \otimes Z_{P},
$$

is an isomorphism.

Proof. By Propositions 2.2 and 2.3, we know that $r_{N K}^{G} r_{K}^{N K}$ is a $P$-monomorphism. Suppose $x \in \Omega_{*}(K)(A K, P K)^{N K / K}$ and $k$ is the index of $K$ in $N K$. Then $r_{K}^{N K} e_{N K}^{K}(x)=k x$, so $x=r_{K}^{N K} e_{N K}^{K}(x / k)$. Since $r_{N K}^{G}$ is a $P$-isomorphism, this implies that $x \otimes 1$ lies in the image of (3).

Now suppose $\mathscr{F}^{\prime}$ and $\mathscr{F}^{\prime \prime}$ are also adjacent, differing by $H$, and suppose $P=P(G: K) \cap P(G: H)$. Recall the bordism exact sequence for a triple of families [8, Proposition 2.2]:

$$
\begin{aligned}
\cdots & \rightarrow \Omega_{*}(G)\left(\mathscr{F}^{\prime}, \mathscr{F}^{\prime \prime}\right) \otimes Z_{P} \stackrel{i_{*}}{\rightarrow} \Omega_{*}(G)\left(\mathscr{F}, \mathscr{F}^{\prime \prime}\right) \otimes Z_{P} \\
& \stackrel{j_{*}}{\rightarrow} \Omega_{*}(G)\left(\mathscr{F}, \mathscr{F}^{\prime}\right) \otimes Z_{P} \rightarrow \cdots
\end{aligned}
$$

Clearly $r_{H}^{G}=r_{H_{*}}^{G} i_{*} \Omega_{*}(G)\left(\mathscr{F}^{\prime}, \mathscr{F}^{\prime \prime}\right) \otimes Z_{P} \rightarrow \Omega_{*}(H)(A H, P H) \otimes Z_{P}$. By Proposition 3.1, $i_{*}$ is a split monomorphism. Similarly, one has that $r_{K}^{G}=r_{K}^{G} j_{*}$, so $r_{H}^{G}+r_{K}^{G}$ is an isomorphism of $\Omega_{*}(G)\left(\mathscr{F}, \mathscr{F}^{\prime}\right) \otimes Z_{P}$ with $\left(\Omega_{*}(H)(A H, P H)^{N H / H} \oplus \Omega_{*}(K)(A K, P K)^{N K / K}\right) \otimes Z_{P}$.

Proposition 3.2. Suppose $G$ is a finite group, $\left(\mathscr{F}, \mathscr{F}^{\prime}\right)$ is a pair of families of subgroups of $G$, and $P=\cap\left\{P(G: K): K \in \mathscr{F}-\mathscr{F}^{\prime}\right\}$. Then the sum of the 
restrictions $r_{K}^{G}$ is an isomorphism,

$$
\Omega_{*}(G)\left(\mathscr{F}, \mathscr{F}^{\prime}\right) \otimes Z_{P} \stackrel{\cong}{\rightarrow} \sum\left\{\Omega_{*}(K)(A K, P K)^{N K / K} \otimes Z_{P}:(K) \subseteq \mathscr{F}-\mathscr{F}^{\prime}\right\} .
$$

The proof, of course, is to repeat the procedure given above. As corollaries, we obtain Theorems 1.1 and 1.2, as well as the following useful result.

Corollary 3.3. Suppose $\left(\mathscr{F}, \mathscr{F}^{\prime}, \mathscr{F}^{\prime \prime}\right)$ is a triple of families, and $P=$ $\cap\left\{P(G: K): K \in \mathscr{F}^{\prime}-\mathscr{F}^{\prime \prime}\right\}$. Then the forgetful homomorphism

$$
i_{*}: \Omega_{*}(G)\left(\mathscr{F}^{\prime}, \mathscr{F}^{\prime \prime}\right) \otimes Z_{P} \rightarrow \Omega_{*}(G)\left(\mathscr{F}, \mathscr{F}^{\prime \prime}\right) \otimes Z_{P}
$$

is a split monomorphism.

Proof. As above, we see that $\left(\Sigma r_{K}^{G}\right) i_{*}=\Sigma r_{K}^{G}$, where the summations are over all $(K) \subset \mathscr{F}^{\prime}$. Thus Proposition 3.2 implies the result.

The virtue of this line of proof is that, so far, it has been absolutely simple-minded, without requiring any knowledge of representation theory or of the classification theory of $G$-vector bundles. We now add the simplest result from the latter area.

PROPOSITION 3.4. Let $G$ be a finite group. If $\Omega_{*}=\Omega_{*}^{U}$, or if $\Omega_{*}=\Omega_{*}^{S O}$ and $G$ has odd order, then $\Omega_{*}(G)(A G, P G)$ is a free $\Omega_{*}$-module on even-dimensional generators. If $G$ has even order, then $\Omega_{*}^{S O}(G)(A G, P G) \otimes Z\left(\frac{1}{2}\right)$ is a free $\Omega_{*}^{S O} \otimes Z\left(\frac{1}{2}\right)$-module on even-dimensional generators.

Proof. The following procedure is well known, but no single reference applies in this generality. We give only an outline.

If $M$ is a manifold with smooth $G$-action, representing an element of $\Omega_{*}(G)(A G, P G)$, then we may replace $M$ by a tubular neighborhood $N$ of the fixed point set $F$ of $G$ in $M$, by [9, Lemma 5.1]. The normal bundle $\nu$ to $F$ in $M$ can be decomposed into $G$-invariant subbundles $\nu_{L}$, indexed by the irreducible real (if $\Omega_{*}=\Omega_{*}^{S O}$ ) or complex (if $\Omega_{*}=\Omega_{*}^{U}$ ) representations $L$ of $G$. This idea goes back to Conner and Floyd [2, Theorem (38.3)]. Next, one may classify the bundle $\nu_{L}$ by a map of $M$ into $B O(j)$, or $B U(j)$, or $B \operatorname{Sp}(j)$. If $\Omega_{*}=\Omega_{*}^{U}$, the $\nu_{L}$ are all complex vector bundles and $B U(j)$ is used; if $\Omega_{*}=\Omega_{*}^{S O}$ the choice is governed by whether the field $\operatorname{Hom}_{G}(L, L)$ of the representation is real, complex, or quaternionic.

If $\Omega_{*}=\Omega_{*}^{U}$, it is now easy to show that $\Omega_{*}^{U}(G)(A G, P G)$ is isomorphic to a direct sum of bordism groups of the form $\Omega_{*}^{U}\left(B U\left(j_{1}\right) \times \cdots \times B U\left(j_{n}\right)\right)$; each of these groups is known to be a free $\Omega_{*}^{U}$-module on even-dimensional generators [3, Lemma (2.3)].

If $\Omega_{*}=\Omega_{*}^{S O}$ and $G$ is of odd order, then some of the representations may be quaternionic. Thus some of the $B U\left(j_{k}\right)$ may be replaced by $B \operatorname{Sp}\left(j_{k}\right)$, but the theorem follows in the same fashion, by use of [2, Theorem (18.1)].

If $\Omega_{*}=\Omega_{*}^{S O}$ and $G$ is of even order the classification scheme must be 
revised, for $F$ need not be oriented. However, we may classify $\nu$ by a map of the (oriented) tubular neighborhood $N$ into an appropriate Thom space. Thus $\Omega_{*}(G)(A G, P G)$ is a sum of bordism groups $\tilde{\Omega}_{*}^{s o}\left(M_{\gamma^{\prime}}\right)$, where $\gamma^{I}=\gamma\left(j_{i}\right)$ $\times \cdots \times \gamma\left(j_{n}\right)$ is a product of universal $j_{k}$-bundles over $B O\left(j_{k}\right)$, or $B U\left(j_{k}\right)$, or $B \operatorname{Sp}\left(j_{k}\right)$, as the case may require. In each case, $\tilde{H}^{*}\left(M \gamma\left(j_{k}\right) ; Z\left(\frac{1}{2}\right)\right)$ is a free $Z\left(\frac{1}{2}\right)$-module on even-dimensional generators. The theorem will now follow, by the Künneth theorem and a lemma of Conner [1, $(3,1)$, p. 93].

By Theorem 1.1 and Proposition 3.4, we obtain the localized freeness theorem.

THeOREM 3.5. If $G$ is a finite group, and $P=P(G)$, then $\Omega_{*}(G) \otimes Z_{P}$ is a free $\Omega_{*} \otimes Z_{P}$-module on even-dimensional generators.

This completes work of Wheeler [11], who proved Theorem 3.5 for $\Omega_{*}^{U}$ and for $\Omega_{*}^{S O}$ and groups of odd order. However, it is not trivial that if $G$ is a finite 2-group, then $\Omega_{*}^{S O}(G) \otimes Z\left(\frac{1}{2}\right)$ is a free $\Omega_{*}^{S O} \otimes Z\left(\frac{1}{2}\right)$-module; indeed, Theorem 3.5 is (so far as I know) the first result on the structure on $\Omega_{*}^{S O}(G)$ that holds for all groups $G$. By methods of Ossa [5], one may improve Theorem 3.5 for abelian groups.

COROLlARY 3.6. If $G$ is a finite abelian group, then $\Omega_{*}^{S O}(G) \otimes Z\left(\frac{1}{2}\right)$ is a free $\Omega_{*}^{S O} \otimes Z\left(\frac{1}{2}\right)$-module on even-dimensional generators.

Again this completes work of Wheeler, who proved Corollary 3.6 for cyclic groups [10].

4. An application to 2-group actions. One of the most difficult problems in equivariant bordism is to compute $\Omega_{*}^{S O}(G)$ in case $G$ is a 2-group. There are essentially no published results, except for the case $G=Z_{2}[1]$, and the theorem of [6] on free actions. Now Theorems 1.1 and 3.5 give us a description of $\Omega_{*}^{S O}(G) \otimes Z\left(\frac{1}{2}\right)$, and in this section we give some information, albeit somewhat crude, about the torsion of $\Omega_{*}^{S O}(G)$.

THEOREM 4.1. Let $G$ be a group of order $2^{k}$. Then a torsion element in $\Omega_{*}^{S O}(G)$ is of order $2^{s}$ for some $s<\frac{1}{2} k(k+3)$. If $G$ is abelian, $s<\frac{1}{2} k(k+1)$.

In other words, the torsion is of bounded order. The specific bounds given may be very excessive, however. In fact there is no known example of an element of order 4 in any $\Omega_{*}^{S O}(G)$, although there is no reason to believe such elements do not exist.

For the proof we shall need the following lemma.

Proposition 4.2. Let $L$ be a subgroup of $G$ of index 2 , and let $\left(\mathcal{F}, \mathcal{F}^{\prime}\right)$ be a pair of G-invariant families of subgroups of $L$. Then the composition $e_{G}^{L} r_{L}^{G}$ is multiplication by two in $\Omega_{*}^{S O}(G)\left(\mathscr{F}, \mathscr{F}^{\prime}\right)$. 
Proof. Let $\chi: G \times M \rightarrow M$ be a smooth action representing an element of $\Omega_{*}^{S O}(G)\left(\mathscr{F}, \mathscr{F}^{\prime}\right)$. Let $\theta$ be the nontrivial one-dimensional real representation of $G$ having kernel $L$. Let $N \subset M$ be a submanifold dual to $\theta$; that is, the normal bundle of $N$ in $M$ is $R \times N$ with action $\theta \times(\chi \mid N)$. Such a submanifold exists by [7, Proposition 4]. Notice that $N$ is orientable, but that the elements of $G-L$ reverse the orientation of $N$. Choose $g_{0} \in G-L$.

Let $M^{\prime}$ be a second copy of $M$, but with action $\chi^{\prime}(g, m)=\chi\left(g_{0} g g_{0}^{-1}, m\right)$. Let $P=N \times[-1,1]$ be a tubular neighborhood of $N$ in $M$, and let $P^{\prime}=N^{\prime}$ $\times[-1,1]$ be the corresponding neighborhood of $N^{\prime}$ in $M^{\prime}$.

Take the disjoint union of $M \times[0,1]$ and $M^{\prime} \times[0,1]$, and identify $(x, t)$ $\in N \times[-1,1]=P \times\{1\}$ with $\left(g_{0} x, t\right) \in N^{\prime} \times[-1,1]=P^{\prime} \times\{0\}$. Call the resulting manifold $Q$.

Now $g_{0} \times 1$ is an orientation-reversing, $G$-equivariant diffeomorphism of $N \times[-1,1]$ with $N^{\prime} \times[-1,1]$. Thus $Q$ is oriented, and inherits an orientation-preserving $G$-action $\chi_{1}$ from $(\chi \times 1)+\left(\chi^{\prime} \times 1\right)$. Also, $N$ separates $M$; in fact, we can obtain $N$ as $f^{-1}\left(S^{m-1}\right)$ for some $G$-map $f: M \rightarrow S^{m} \subset E Z_{2}$ (compare $\$ 2$ above). Hence $M$ is the union of $L$-invariant submanifolds in the form $M=V \cup_{h} V^{\prime}$, where $\partial V=N$ and $h: N \rightarrow N^{\prime}$ is left multiplication by $g_{0}$. It is then easy to see that

$$
\partial\left(Q, \chi_{1}\right)=e_{G}^{L} r_{L}^{G}(M, \chi)-(M, \chi)-\left(M^{\prime}, \chi^{\prime}\right) .
$$

Since $\left[M^{\prime}, \chi\right]=[M, \chi] \in \Omega_{*}(G)\left(\mathscr{F}, \mathscr{F}^{\prime}\right)$ by $[2, \S 20]$, this implies the proposition.

We can now prove Theorem 4.1. For $0 \leqslant j \leqslant k$, define families

$$
\mathscr{F}_{j}=\left\{H<G: \text { order of } H<2^{j}\right\} .
$$

Thus $\Omega_{*}(G)\left(\mathscr{F}_{k}\right)=\Omega_{*}(G)(A G)$, while $\Omega_{*}(G)\left(\mathscr{F}_{0}\right)=\hat{\Omega}_{*}(G)$, the bordism of free $G$-actions. Since all elements of $\mathscr{F}_{j}-\mathscr{F}_{j-1}$ are maximal in $\mathscr{F}_{j}$, it follows from [9, Corollary 5.2, p. 21] that

$$
\Omega_{*}(G)\left(\mathscr{F}_{j}, \mathscr{F}_{j-1}\right) \cong \sum\left\{\Omega_{*}(N K)(A K, P K):(K) \subseteq \mathscr{F}_{j}-\mathscr{F}_{j-1}\right\} .
$$

Next we shall show that if $H$ is a normal subgroup of $G$ of index $2^{i}$, then all torsion of $\Omega_{*}(G)(A H, P H)$ is of exponent $2^{i+1}$. This is done by induction on $i$. If $i=0$ (that is, if $H=G$ ), then, as in the proof of Proposition 3.4, we write $\Omega_{*}(G)(A G, P G)$ as a sum of reduced bordism groups of Thom spaces. One may check that $\tilde{H}_{*}(M \gamma(j))$ has no torsion except of order two; by [2, Theorem (15.2)] the same is true for $\Omega_{*}(G)(A G, P G)$.

Now if $i>0$, then $H$ is a proper subgroup of $G$. We may choose a normal subgroup $L$ of index two in $G$, such that $L$ contains $H$. Suppose $x \in$ $\Omega_{*}(G)(A H, P H)$ is a torsion class. Then $2^{i} r_{L}^{G}(x)=0$ by inductive assumption. By Theorem 4.1,

$$
2^{i+1} x=2^{i} e_{G}^{L} r_{L}^{G}(x)=0 .
$$


From (4) we now see that the torsion classes in $\Omega_{*}(G)\left(\mathscr{F}_{j}, \mathscr{F}_{j-1}\right)$ are of exponent $2^{(k-j)+1}$. Consider now the exact sequences

$$
\ldots \rightarrow \Omega_{*}(G)\left(\mathscr{F}_{j}, \mathscr{F}_{j-1}\right) \stackrel{r_{*}}{\rightarrow} \Omega_{*}(G)\left(A G, \mathscr{F}_{j-1}\right) \rightarrow \Omega_{*}(G)\left(A G, \mathscr{F}_{j}\right) \rightarrow \ldots
$$

The crucial observation is that $r_{*}$ is a 2-monomorphism, by Corollary 3.3. Therefore if $y=r_{*} x$ is a torsion class we may assume $x$ is a torsion class. An easy inductive argument shows that all torsion in $\Omega_{*}(G)\left(\mathscr{F}_{k}, \mathscr{F}_{0}\right)$ is of exponent $2^{n}$, where $n=\Sigma_{j}((k-j)+1)=\frac{1}{2} k(k+1)$.

If $G$ is abelian, then by [6] we know that $\Omega_{*}(G)\left(\mathscr{F}_{0}\right) \rightarrow \Omega_{*}(G)\left(\mathscr{F}_{k}\right)$ has torsionfree image. This finishes the proof in that case. In general, it follows from [2, Theorem (15.2)] that all torsion in $\hat{\Omega}_{*}(G)=\Omega_{*}(G)\left(\mathscr{F}_{0}\right)$ is of exponent $2^{k}$, and hence that the torsion in $\Omega_{*}(G)=\Omega_{*}(G)\left(\mathscr{F}_{k}\right)$ is of exponent $2^{s}$ for $s=k+\frac{1}{2} k(k+1)=\frac{1}{2} k(k+3)$. Thus the proof of Theorem 4.1 is complete.

\section{REFERENCES}

1. P. E. Conner, Lectures on the action of a finite group, Lecture Notes in Math., vol. 73, Springer-Verlag, Berlin, 1968.

2. P. E. Conner and E. E. Floyd, Differentiable periodic maps, Ergebnisse der Mathematik und ihrer Grenzgebiete, Band 33, Springer-Verlag, Berlin, 1964.

3. P. E. Conner and L. Smith, On the complex bordism of finite complexes, Inst. Hautes Études Sci. Publ. Math. 37 (1969), 117-222.

4. J. Milnor, Construction of universal bundles, I, Ann. of Math. (2) 63 (1956), 272-284.

5. E. Ossa, Unitary bordism of abelian groups, Proc. Amer. Math. Soc. 33 (1972), 568-571.

6. R. J. Rowlett, Bounding a free action of an abelian group, Duke Math. J. 41 (1974), 381-385. 7. Torsion in the bordism of oriented involutions, Trans. Amer. Math. Soc. 231 (1977), 541-548.

8. R. E. Stong, Complex and oriented equivariant bordism, Topology of Manifolds (Proc. Inst., Univ. of Georgia, Athens, 1969), Markham, Chicago, 1970, pp. 291-316.

9. , Unoriented bordism and actions of finite groups, Mem. Amer. Math. Soc. No. 103 (1970).

10. E. Wheeler, Oriented bordism of cyclic group actions, Thesis, Univ. of Virginia, Charlottesville, 1973.

11. , Localizing equivariant bordism, Proc. Amer. Math. Soc. 44 (1974), 485-491.

Department of Mathematics, University of Tennessee, Knoxville, Tennessee 37916 